\title{
Partial Differentiation on Normed Linear Spaces $\mathcal{R}^{n}$
}

\author{
Noboru Endou \\ Gifu National College of Technology \\ Japan \\ Keiichi Miyajima \\ Ibaraki University \\ Hitachi, Japan
}

Yasunari Shidama

Shinshu University

Nagano, Japan

\begin{abstract}
Summary. In this article, we define the partial differentiation of functions of real variable and prove the linearity of this operator [18].
\end{abstract}

MML identifier: PDIFF_1, version: 7.8.05 4.84.971

The notation and terminology used here are introduced in the following papers: $[21],[24],[25],[5],[26],[7],[6],[15],[13],[3],[1],[20],[11],[22],[23],[14],[8]$, $[2],[4],[27],[28],[16],[9],[19],[17],[12]$, and [10].

\section{Preliminaries}

Let $i, n$ be elements of $\mathbb{N}$. The functor $\operatorname{proj}(i, n)$ yielding a function from $\mathcal{R}^{n}$ into $\mathbb{R}$ is defined by:

(Def. 1) For every element $x$ of $\mathcal{R}^{n}$ holds $(\operatorname{proj}(i, n))(x)=x(i)$.

Next we state two propositions:

(1) $\quad \operatorname{dom} \operatorname{proj}(1,1)=\mathcal{R}^{1}$ and $\operatorname{rng} \operatorname{proj}(1,1)=\mathbb{R}$ and for every element $x$ of $\mathbb{R}$ holds $(\operatorname{proj}(1,1))(\langle x\rangle)=x$ and $(\operatorname{proj}(1,1))^{-1}(x)=\langle x\rangle$.

(2)(i) $\quad(\operatorname{proj}(1,1))^{-1}$ is a function from $\mathbb{R}$ into $\mathcal{R}^{1}$,

(ii) $(\operatorname{proj}(1,1))^{-1}$ is one-to-one,

(iii) $\operatorname{dom}\left((\operatorname{proj}(1,1))^{-1}\right)=\mathbb{R}$,

(iv) $\operatorname{rng}\left((\operatorname{proj}(1,1))^{-1}\right)=\mathcal{R}^{1}$, and 
(v) there exists a function $g$ from $\mathbb{R}$ into $\mathcal{R}^{1}$ such that $g$ is bijective and $(\operatorname{proj}(1,1))^{-1}=g$.

One can check that $\operatorname{proj}(1,1)$ is bijective.

Let $g$ be a partial function from $\mathbb{R}$ to $\mathbb{R}$. The functor $\langle g\rangle$ yields a partial function from $\mathcal{R}^{1}$ to $\mathcal{R}^{1}$ and is defined as follows:

(Def. 2) $\langle g\rangle=(\operatorname{proj}(1,1))^{-1} \cdot g \cdot \operatorname{proj}(1,1)$.

Let $n$ be an element of $\mathbb{N}$ and let $g$ be a partial function from $\mathcal{R}^{n}$ to $\mathbb{R}$. The functor $\langle g\rangle$ yielding a partial function from $\mathcal{R}^{n}$ to $\mathcal{R}^{1}$ is defined as follows:

(Def. 3) $\langle g\rangle=(\operatorname{proj}(1,1))^{-1} \cdot g$.

Let $i, n$ be elements of $\mathbb{N}$. The functor $\operatorname{Proj}(i, n)$ yielding a function from $\left\langle\mathcal{E}^{n},\|\cdot\|\right\rangle$ into $\left\langle\mathcal{E}^{1},\|\cdot\|\right\rangle$ is defined as follows:

(Def. 4) For every point $x$ of $\left\langle\mathcal{E}^{n},\|\cdot\|\right\rangle$ holds $(\operatorname{Proj}(i, n))(x)=\langle(\operatorname{proj}(i, n))(x)\rangle$.

Let $i$ be an element of $\mathbb{N}$ and let $x$ be a finite sequence of elements of $\mathbb{R}$. The functor $\operatorname{reproj}(i, x)$ yielding a function is defined as follows:

(Def. 5) dom $\operatorname{reproj}(i, x)=\mathbb{R}$ and for every element $r$ of $\mathbb{R}$ holds $(\operatorname{reproj}(i, x))(r)=\operatorname{Replace}(x, i, r)$.

Let $n, i$ be elements of $\mathbb{N}$ and let $x$ be an element of $\mathcal{R}^{n}$. Then $\operatorname{reproj}(i, x)$ is a function from $\mathbb{R}$ into $\mathcal{R}^{n}$.

Let $n, i$ be elements of $\mathbb{N}$ and let $x$ be a point of $\left\langle\mathcal{E}^{n},\|\cdot\|\right\rangle$. The functor $\operatorname{reproj}(i, x)$ yielding a function from $\left\langle\mathcal{E}^{1},\|\cdot\|\right\rangle$ into $\left\langle\mathcal{E}^{n},\|\cdot\|\right\rangle$ is defined by the condition (Def. 6).

(Def. 6) Let $r$ be an element of $\left\langle\mathcal{E}^{1},\|\cdot\|\right\rangle$. Then there exists an element $q$ of $\mathbb{R}$ and there exists an element $y$ of $\mathcal{R}^{n}$ such that $r=\langle q\rangle$ and $y=x$ and $(\operatorname{reproj}(i, x))(r)=(\operatorname{reproj}(i, y))(q)$.

Let $m, n$ be non empty elements of $\mathbb{N}$, let $f$ be a partial function from $\mathcal{R}^{m}$ to $\mathcal{R}^{n}$, and let $x$ be an element of $\mathcal{R}^{m}$. We say that $f$ is differentiable in $x$ if and only if the condition (Def. 7) is satisfied.

(Def. 7) There exists a partial function $g$ from $\left\langle\mathcal{E}^{m},\|\cdot\|\right\rangle$ to $\left\langle\mathcal{E}^{n},\|\cdot\|\right\rangle$ and there exists a point $y$ of $\left\langle\mathcal{E}^{m},\|\cdot\|\right\rangle$ such that $f=g$ and $x=y$ and $g$ is differentiable in $y$.

Let $m, n$ be non empty elements of $\mathbb{N}$, let $f$ be a partial function from $\mathcal{R}^{m}$ to $\mathcal{R}^{n}$, and let $x$ be an element of $\mathcal{R}^{m}$. Let us assume that $f$ is differentiable in $x$. The functor $f^{\prime}(x)$ yields a function from $\mathcal{R}^{m}$ into $\mathcal{R}^{n}$ and is defined as follows:

(Def. 8) There exists a partial function $g$ from $\left\langle\mathcal{E}^{m},\|\cdot\|\right\rangle$ to $\left\langle\mathcal{E}^{n},\|\cdot\|\right\rangle$ and there exists a point $y$ of $\left\langle\mathcal{E}^{m},\|\cdot\|\right\rangle$ such that $f=g$ and $x=y$ and $f^{\prime}(x)=g^{\prime}(y)$.

We now state four propositions:

(3) Let $I$ be a function from $\mathbb{R}$ into $\mathcal{R}^{1}$. Suppose $I=(\operatorname{proj}(1,1))^{-1}$. Then 
(i) for every vector $x$ of $\left\langle\mathcal{E}^{1},\|\cdot\|\right\rangle$ and for every element $y$ of $\mathbb{R}$ such that $x=I(y)$ holds $\|x\|=|y|$,

(ii) for all vectors $x, y$ of $\left\langle\mathcal{E}^{1},\|\cdot\|\right\rangle$ and for all elements $a, b$ of $\mathbb{R}$ such that $x=I(a)$ and $y=I(b)$ holds $x+y=I(a+b)$,

(iii) for every vector $x$ of $\left\langle\mathcal{E}^{1},\|\cdot\|\right\rangle$ and for every element $y$ of $\mathbb{R}$ and for every real number $a$ such that $x=I(y)$ holds $a \cdot x=I(a \cdot y)$,

(iv) for every vector $x$ of $\left\langle\mathcal{E}^{1},\|\cdot\|\right\rangle$ and for every element $a$ of $\mathbb{R}$ such that $x=I(a)$ holds $-x=I(-a)$, and

(v) for all vectors $x, y$ of $\left\langle\mathcal{E}^{1},\|\cdot\|\right\rangle$ and for all elements $a, b$ of $\mathbb{R}$ such that $x=I(a)$ and $y=I(b)$ holds $x-y=I(a-b)$.

(4) Let $J$ be a function from $\mathcal{R}^{1}$ into $\mathbb{R}$. Suppose $J=\operatorname{proj}(1,1)$. Then

(i) for every vector $x$ of $\left\langle\mathcal{E}^{1},\|\cdot\|\right\rangle$ and for every element $y$ of $\mathbb{R}$ such that $J(x)=y$ holds $\|x\|=|y|$,

(ii) for all vectors $x, y$ of $\left\langle\mathcal{E}^{1},\|\cdot\|\right\rangle$ and for all elements $a, b$ of $\mathbb{R}$ such that $J(x)=a$ and $J(y)=b$ holds $J(x+y)=a+b$,

(iii) for every vector $x$ of $\left\langle\mathcal{E}^{1},\|\cdot\|\right\rangle$ and for every element $y$ of $\mathbb{R}$ and for every real number $a$ such that $J(x)=y$ holds $J(a \cdot x)=a \cdot y$,

(iv) for every vector $x$ of $\left\langle\mathcal{E}^{1},\|\cdot\|\right\rangle$ and for every element $a$ of $\mathbb{R}$ such that $J(x)=a$ holds $J(-x)=-a$, and

(v) for all vectors $x, y$ of $\left\langle\mathcal{E}^{1},\|\cdot\|\right\rangle$ and for all elements $a, b$ of $\mathbb{R}$ such that $J(x)=a$ and $J(y)=b$ holds $J(x-y)=a-b$.

(5) Let $I$ be a function from $\mathbb{R}$ into $\mathcal{R}^{1}$ and $J$ be a function from $\mathcal{R}^{1}$ into $\mathbb{R}$. Suppose $I=(\operatorname{proj}(1,1))^{-1}$ and $J=\operatorname{proj}(1,1)$. Then

(i) for every rest $R$ of $\left\langle\mathcal{E}^{1},\|\cdot\|\right\rangle,\left\langle\mathcal{E}^{1},\|\cdot\|\right\rangle$ holds $J \cdot R \cdot I$ is a rest, and

(ii) for every linear operator $L$ from $\left\langle\mathcal{E}^{1},\|\cdot\|\right\rangle$ into $\left\langle\mathcal{E}^{1},\|\cdot\|\right\rangle$ holds $J \cdot L \cdot I$ is a linear function.

(6) Let $I$ be a function from $\mathbb{R}$ into $\mathcal{R}^{1}$ and $J$ be a function from $\mathcal{R}^{1}$ into $\mathbb{R}$. Suppose $I=(\operatorname{proj}(1,1))^{-1}$ and $J=\operatorname{proj}(1,1)$. Then

(i) for every rest $R$ holds $I \cdot R \cdot J$ is a rest of $\left\langle\mathcal{E}^{1},\|\cdot\|\right\rangle,\left\langle\mathcal{E}^{1},\|\cdot\|\right\rangle$, and

(ii) for every linear function $L$ holds $I \cdot L \cdot J$ is a bounded linear operator from $\left\langle\mathcal{E}^{1},\|\cdot\|\right\rangle$ into $\left\langle\mathcal{E}^{1},\|\cdot\|\right\rangle$.

In the sequel $f$ is a partial function from $\left\langle\mathcal{E}^{1},\|\cdot\|\right\rangle$ to $\left\langle\mathcal{E}^{1},\|\cdot\|\right\rangle, g$ is a partial function from $\mathbb{R}$ to $\mathbb{R}, x$ is a point of $\left\langle\mathcal{E}^{1},\|\cdot\|\right\rangle$, and $y$ is an element of $\mathbb{R}$.

We now state four propositions:

(7) If $f=\langle g\rangle$ and $x=\langle y\rangle$ and $f$ is differentiable in $x$, then $g$ is differentiable in $y$ and $g^{\prime}(y)=\left(\operatorname{proj}(1,1) \cdot f^{\prime}(x) \cdot(\operatorname{proj}(1,1))^{-1}\right)(1)$.

(8) If $f=\langle g\rangle$ and $x=\langle y\rangle$ and $g$ is differentiable in $y$, then $f$ is differentiable in $x$ and $f^{\prime}(x)(\langle 1\rangle)=\left\langle g^{\prime}(y)\right\rangle$.

(9) If $f=\langle g\rangle$ and $x=\langle y\rangle$, then $f$ is differentiable in $x$ iff $g$ is differentiable in $y$. 
(10) If $f=\langle g\rangle$ and $x=\langle y\rangle$ and $f$ is differentiable in $x$, then $f^{\prime}(x)(\langle 1\rangle)=$ $\left\langle g^{\prime}(y)\right\rangle$.

\section{Partial Differentiation}

For simplicity, we adopt the following rules: $m, n$ are non empty elements of $\mathbb{N}, i, j$ are elements of $\mathbb{N}, f$ is a partial function from $\left\langle\mathcal{E}^{n},\|\cdot\|\right\rangle$ to $\left\langle\mathcal{E}^{1},\|\cdot\|\right\rangle, g$ is a partial function from $\mathcal{R}^{n}$ to $\mathbb{R}, x$ is a point of $\left\langle\mathcal{E}^{n},\|\cdot\|\right\rangle$, and $y$ is an element of $\mathcal{R}^{n}$.

Let $n, m$ be non empty elements of $\mathbb{N}$, let $i$ be an element of $\mathbb{N}$, let $f$ be a partial function from $\left\langle\mathcal{E}^{m},\|\cdot\|\right\rangle$ to $\left\langle\mathcal{E}^{n},\|\cdot\|\right\rangle$, and let $x$ be a point of $\left\langle\mathcal{E}^{m},\|\cdot\|\right\rangle$. We say that $f$ is partially differentiable in $x$ w.r.t. $i$ if and only if:

(Def. 9) $f \cdot \operatorname{reproj}(i, x)$ is differentiable in $(\operatorname{Proj}(i, m))(x)$.

Let $m, n$ be non empty elements of $\mathbb{N}$, let $i$ be an element of $\mathbb{N}$, let $f$ be a partial function from $\left\langle\mathcal{E}^{m},\|\cdot\|\right\rangle$ to $\left\langle\mathcal{E}^{n},\|\cdot\|\right\rangle$, and let $x$ be a point of $\left\langle\mathcal{E}^{m},\|\cdot\|\right\rangle$. The functor partdiff $(f, x, i)$ yielding a point of the real norm space of bounded linear operators from $\left\langle\mathcal{E}^{1},\|\cdot\|\right\rangle$ into $\left\langle\mathcal{E}^{n},\|\cdot\|\right\rangle$ is defined as follows:

(Def. 10) $\operatorname{partdiff}(f, x, i)=(f \cdot \operatorname{reproj}(i, x))^{\prime}((\operatorname{Proj}(i, m))(x))$.

Let $n$ be a non empty element of $\mathbb{N}$, let $i$ be an element of $\mathbb{N}$, let $f$ be a partial function from $\mathcal{R}^{n}$ to $\mathbb{R}$, and let $x$ be an element of $\mathcal{R}^{n}$. We say that $f$ is partially differentiable in $x$ w.r.t. $i$ if and only if:

(Def. 11) $f \cdot \operatorname{reproj}(i, x)$ is differentiable in $(\operatorname{proj}(i, n))(x)$.

Let $n$ be a non empty element of $\mathbb{N}$, let $i$ be an element of $\mathbb{N}$, let $f$ be a partial function from $\mathcal{R}^{n}$ to $\mathbb{R}$, and let $x$ be an element of $\mathcal{R}^{n}$. The functor partdiff $(f, x, i)$ yields a real number and is defined by:

(Def. 12) $\operatorname{partdiff}(f, x, i)=(f \cdot \operatorname{reproj}(i, x))^{\prime}((\operatorname{proj}(i, n))(x))$.

We now state several propositions:

(11) $\operatorname{Proj}(i, n)=(\operatorname{proj}(1,1))^{-1} \cdot \operatorname{proj}(i, n)$.

(12) If $x=y$, then $\operatorname{reproj}(i, y) \cdot \operatorname{proj}(1,1)=\operatorname{reproj}(i, x)$.

(13) If $f=\langle g\rangle$ and $x=y$, then $\langle g \cdot \operatorname{reproj}(i, y)\rangle=f \cdot \operatorname{reproj}(i, x)$.

(14) Suppose $f=\langle g\rangle$ and $x=y$. Then $f$ is partially differentiable in $x$ w.r.t. $i$ if and only if $g$ is partially differentiable in $y$ w.r.t. $i$.

(15) If $f=\langle g\rangle$ and $x=y$ and $f$ is partially differentiable in $x$ w.r.t. $i$, then $(\operatorname{partdiff}(f, x, i))(\langle 1\rangle)=\langle\operatorname{partdiff}(g, y, i)\rangle$.

Let $m, n$ be non empty elements of $\mathbb{N}$, let $i$ be an element of $\mathbb{N}$, let $f$ be a partial function from $\mathcal{R}^{m}$ to $\mathcal{R}^{n}$, and let $x$ be an element of $\mathcal{R}^{m}$. We say that $f$ is partially differentiable in $x$ w.r.t. $i$ if and only if the condition (Def. 13) is satisfied. 
(Def. 13) There exists a partial function $g$ from $\left\langle\mathcal{E}^{m},\|\cdot\|\right\rangle$ to $\left\langle\mathcal{E}^{n},\|\cdot\|\right\rangle$ and there exists a point $y$ of $\left\langle\mathcal{E}^{m},\|\cdot\|\right\rangle$ such that $f=g$ and $x=y$ and $g$ is partially differentiable in $y$ w.r.t. $i$.

Let $m, n$ be non empty elements of $\mathbb{N}$, let $i$ be an element of $\mathbb{N}$, let $f$ be a partial function from $\mathcal{R}^{m}$ to $\mathcal{R}^{n}$, and let $x$ be an element of $\mathcal{R}^{m}$. Let us assume that $f$ is partially differentiable in $x$ w.r.t. $i$. The functor $\operatorname{partdiff}(f, x, i)$ yielding an element of $\mathcal{R}^{n}$ is defined as follows:

(Def. 14) There exists a partial function $g$ from $\left\langle\mathcal{E}^{m},\|\cdot\|\right\rangle$ to $\left\langle\mathcal{E}^{n},\|\cdot\|\right\rangle$ and there exists a point $y$ of $\left\langle\mathcal{E}^{m},\|\cdot\|\right\rangle$ such that $f=g$ and $x=y$ and $\operatorname{partdiff}(f, x, i)=(\operatorname{partdiff}(g, y, i))(\langle 1\rangle)$.

One can prove the following four propositions:

(16) Let $m, n$ be non empty elements of $\mathbb{N}, F$ be a partial function from $\left\langle\mathcal{E}^{m},\|\cdot\|\right\rangle$ to $\left\langle\mathcal{E}^{n},\|\cdot\|\right\rangle, G$ be a partial function from $\mathcal{R}^{m}$ to $\mathcal{R}^{n}, x$ be a point of $\left\langle\mathcal{E}^{m},\|\cdot\|\right\rangle$, and $y$ be an element of $\mathcal{R}^{m}$. Suppose $F=G$ and $x=y$. Then $F$ is partially differentiable in $x$ w.r.t. $i$ if and only if $G$ is partially differentiable in $y$ w.r.t. $i$.

(17) Let $m, n$ be non empty elements of $\mathbb{N}, F$ be a partial function from $\left\langle\mathcal{E}^{m},\|\cdot\|\right\rangle$ to $\left\langle\mathcal{E}^{n},\|\cdot\|\right\rangle, G$ be a partial function from $\mathcal{R}^{m}$ to $\mathcal{R}^{n}, x$ be a point of $\left\langle\mathcal{E}^{m},\|\cdot\|\right\rangle$, and $y$ be an element of $\mathcal{R}^{m}$. Suppose $F=G$ and $x=y$ and $F$ is partially differentiable in $x$ w.r.t. $i$. Then $(\operatorname{partdiff}(F, x, i))(\langle 1\rangle)=$ partdiff $(G, y, i)$.

(18) Let $g_{1}$ be a partial function from $\mathcal{R}^{n}$ to $\mathcal{R}^{1}$. Suppose $g_{1}=\langle g\rangle$. Then $g_{1}$ is partially differentiable in $y$ w.r.t. $i$ if and only if $g$ is partially differentiable in $y$ w.r.t. $i$.

(19) Let $g_{1}$ be a partial function from $\mathcal{R}^{n}$ to $\mathcal{R}^{1}$. Suppose $g_{1}=\langle g\rangle$ and $g_{1}$ is partially differentiable in $y$ w.r.t. $i$. Then $\operatorname{partdiff}\left(g_{1}, y, i\right)=$ $\langle\operatorname{partdiff}(g, y, i)\rangle$.

\section{Linearity of Partial Differential Operator}

For simplicity, we use the following convention: $X$ is a set, $r$ is a real number, $f, f_{1}, f_{2}$ are partial functions from $\left\langle\mathcal{E}^{m},\|\cdot\|\right\rangle$ to $\left\langle\mathcal{E}^{n},\|\cdot\|\right\rangle, g, g_{1}, g_{2}$ are partial functions from $\mathcal{R}^{n}$ to $\mathbb{R}, h$ is a partial function from $\mathcal{R}^{m}$ to $\mathcal{R}^{n}, x$ is a point of $\left\langle\mathcal{E}^{m},\|\cdot\|\right\rangle, y$ is an element of $\mathcal{R}^{n}$, and $z$ is an element of $\mathcal{R}^{m}$.

Let $m, n$ be non empty elements of $\mathbb{N}$, let $i, j$ be elements of $\mathbb{N}$, let $f$ be a partial function from $\left\langle\mathcal{E}^{m},\|\cdot\|\right\rangle$ to $\left\langle\mathcal{E}^{n},\|\cdot\|\right\rangle$, and let $x$ be a point of $\left\langle\mathcal{E}^{m},\|\cdot\|\right\rangle$. We say that $f$ is partially differentiable in $x$ w.r.t. $i$ and $j$ if and only if:

(Def. 15) $\operatorname{Proj}(j, n) \cdot f \cdot \operatorname{reproj}(i, x)$ is differentiable in $(\operatorname{Proj}(i, m))(x)$.

Let $m, n$ be non empty elements of $\mathbb{N}$, let $i, j$ be elements of $\mathbb{N}$, let $f$ be a partial function from $\left\langle\mathcal{E}^{m},\|\cdot\|\right\rangle$ to $\left\langle\mathcal{E}^{n},\|\cdot\|\right\rangle$, and let $x$ be a point of $\left\langle\mathcal{E}^{m},\|\cdot\|\right\rangle$. 
The functor partdiff $(f, x, i, j)$ yields a point of the real norm space of bounded linear operators from $\left\langle\mathcal{E}^{1},\|\cdot\|\right\rangle$ into $\left\langle\mathcal{E}^{1},\|\cdot\|\right\rangle$ and is defined by:

(Def. 16) $\quad \operatorname{partdiff}(f, x, i, j)=(\operatorname{Proj}(j, n) \cdot f \cdot \operatorname{reproj}(i, x))^{\prime}((\operatorname{Proj}(i, m))(x))$.

Let $m, n$ be non empty elements of $\mathbb{N}$, let $i, j$ be elements of $\mathbb{N}$, let $h$ be a partial function from $\mathcal{R}^{m}$ to $\mathcal{R}^{n}$, and let $z$ be an element of $\mathcal{R}^{m}$. We say that $h$ is partially differentiable in $z$ w.r.t. $i$ and $j$ if and only if:

(Def. 17) $\operatorname{proj}(j, n) \cdot h \cdot \operatorname{reproj}(i, z)$ is differentiable in $(\operatorname{proj}(i, m))(z)$.

Let $m, n$ be non empty elements of $\mathbb{N}$, let $i, j$ be elements of $\mathbb{N}$, let $h$ be a partial function from $\mathcal{R}^{m}$ to $\mathcal{R}^{n}$, and let $z$ be an element of $\mathcal{R}^{m}$. The functor partdiff $(h, z, i, j)$ yielding a real number is defined as follows:

(Def. 18) $\operatorname{partdiff}(h, z, i, j)=(\operatorname{proj}(j, n) \cdot h \cdot \operatorname{reproj}(i, z))^{\prime}((\operatorname{proj}(i, m))(z))$.

The following propositions are true:

(20) Let $m, n$ be non empty elements of $\mathbb{N}, F$ be a partial function from $\left\langle\mathcal{E}^{m},\|\cdot\|\right\rangle$ to $\left\langle\mathcal{E}^{n},\|\cdot\|\right\rangle, G$ be a partial function from $\mathcal{R}^{m}$ to $\mathcal{R}^{n}, x$ be a point of $\left\langle\mathcal{E}^{m},\|\cdot\|\right\rangle$, and $y$ be an element of $\mathcal{R}^{m}$. Suppose $F=G$ and $x=y$. Then $F$ is differentiable in $x$ if and only if $G$ is differentiable in $y$.

(21) Let $m, n$ be non empty elements of $\mathbb{N}, F$ be a partial function from $\left\langle\mathcal{E}^{m},\|\cdot\|\right\rangle$ to $\left\langle\mathcal{E}^{n},\|\cdot\|\right\rangle, G$ be a partial function from $\mathcal{R}^{m}$ to $\mathcal{R}^{n}, x$ be a point of $\left\langle\mathcal{E}^{m},\|\cdot\|\right\rangle$, and $y$ be an element of $\mathcal{R}^{m}$. If $F=G$ and $x=y$ and $F$ is differentiable in $x$, then $F^{\prime}(x)=G^{\prime}(y)$.

(22) If $f=h$ and $x=z$, then $\operatorname{Proj}(j, n) \cdot f \cdot \operatorname{reproj}(i, x)=\langle\operatorname{proj}(j, n) \cdot h$. $\operatorname{reproj}(i, z)\rangle$.

(23) Suppose $f=h$ and $x=z$. Then $f$ is partially differentiable in $x$ w.r.t. $i$ and $j$ if and only if $h$ is partially differentiable in $z$ w.r.t. $i$ and $j$.

(24) If $f=h$ and $x=z$ and $f$ is partially differentiable in $x$ w.r.t. $i$ and $j$, then $(\operatorname{partdiff}(f, x, i, j))(\langle 1\rangle)=\langle\operatorname{partdiff}(h, z, i, j)\rangle$.

Let $m, n$ be non empty elements of $\mathbb{N}$, let $i$ be an element of $\mathbb{N}$, let $f$ be a partial function from $\left\langle\mathcal{E}^{m},\|\cdot\|\right\rangle$ to $\left\langle\mathcal{E}^{n},\|\cdot\|\right\rangle$, and let $X$ be a set. We say that $f$ is partially differentiable on $X$ w.r.t. $i$ if and only if:

(Def. 19) $X \subseteq \operatorname{dom} f$ and for every point $x$ of $\left\langle\mathcal{E}^{m},\|\cdot\|\right\rangle$ such that $x \in X$ holds $f \nmid X$ is partially differentiable in $x$ w.r.t. $i$.

We now state the proposition

(25) If $f$ is partially differentiable on $X$ w.r.t. $i$, then $X$ is a subset of $\left\langle\mathcal{E}^{m},\|\cdot\|\right\rangle$.

Let $m, n$ be non empty elements of $\mathbb{N}$, let $i$ be an element of $\mathbb{N}$, let $f$ be a partial function from $\left\langle\mathcal{E}^{m},\|\cdot\|\right\rangle$ to $\left\langle\mathcal{E}^{n},\|\cdot\|\right\rangle$, and let us consider $X$. Let us assume that $f$ is partially differentiable on $X$ w.r.t. $i$. The functor $f \uparrow^{i} X$ yielding a partial function from $\left\langle\mathcal{E}^{m},\|\cdot\|\right\rangle$ to the real norm space of bounded linear operators from $\left\langle\mathcal{E}^{1},\|\cdot\|\right\rangle$ into $\left\langle\mathcal{E}^{n},\|\cdot\|\right\rangle$ is defined by: 
(Def. 20) $\operatorname{dom}\left(f \uparrow^{i} X\right)=X$ and for every point $x$ of $\left\langle\mathcal{E}^{m},\|\cdot\|\right\rangle$ such that $x \in X$ holds $\left(f \uparrow^{i} X\right)_{x}=\operatorname{partdiff}(f, x, i)$.

The following propositions are true:

(26) $\left(f_{1}+f_{2}\right) \cdot \operatorname{reproj}(i, x)=f_{1} \cdot \operatorname{reproj}(i, x)+f_{2} \cdot \operatorname{reproj}(i, x)$ and $\left(f_{1}-f_{2}\right) \cdot$ $\operatorname{reproj}(i, x)=f_{1} \cdot \operatorname{reproj}(i, x)-f_{2} \cdot \operatorname{reproj}(i, x)$.

(27) $\quad r(f \cdot \operatorname{reproj}(i, x))=(r f) \cdot \operatorname{reproj}(i, x)$.

(28) Suppose $f_{1}$ is partially differentiable in $x$ w.r.t. $i$ and $f_{2}$ is partially differentiable in $x$ w.r.t. $i$. Then $f_{1}+f_{2}$ is partially differentiable in $x$ w.r.t. $i$ and partdiff $\left(f_{1}+f_{2}, x, i\right)=\operatorname{partdiff}\left(f_{1}, x, i\right)+\operatorname{partdiff}\left(f_{2}, x, i\right)$.

(29) Suppose $g_{1}$ is partially differentiable in $y$ w.r.t. $i$ and $g_{2}$ is partially differentiable in $y$ w.r.t. $i$. Then $g_{1}+g_{2}$ is partially differentiable in $y$ w.r.t. $i$ and partdiff $\left(g_{1}+g_{2}, y, i\right)=\operatorname{partdiff}\left(g_{1}, y, i\right)+\operatorname{partdiff}\left(g_{2}, y, i\right)$.

(30) Suppose $f_{1}$ is partially differentiable in $x$ w.r.t. $i$ and $f_{2}$ is partially differentiable in $x$ w.r.t. $i$. Then $f_{1}-f_{2}$ is partially differentiable in $x$ w.r.t. $i$ and partdiff $\left(f_{1}-f_{2}, x, i\right)=\operatorname{partdiff}\left(f_{1}, x, i\right)-\operatorname{partdiff}\left(f_{2}, x, i\right)$.

(31) Suppose $g_{1}$ is partially differentiable in $y$ w.r.t. $i$ and $g_{2}$ is partially differentiable in $y$ w.r.t. $i$. Then $g_{1}-g_{2}$ is partially differentiable in $y$ w.r.t. $i$ and partdiff $\left(g_{1}-g_{2}, y, i\right)=\operatorname{partdiff}\left(g_{1}, y, i\right)-\operatorname{partdiff}\left(g_{2}, y, i\right)$.

(32) Suppose $f$ is partially differentiable in $x$ w.r.t. $i$. Then $r f$ is partially differentiable in $x$ w.r.t. $i$ and partdiff $(r f, x, i)=r \cdot \operatorname{partdiff}(f, x, i)$.

(33) Suppose $g$ is partially differentiable in $y$ w.r.t. $i$. Then $r g$ is partially differentiable in $y$ w.r.t. $i$ and partdiff $(r g, y, i)=r \cdot \operatorname{partdiff}(g, y, i)$.

\section{REFERENCES}

[1] Grzegorz Bancerek. The ordinal numbers. Formalized Mathematics, 1(1):91-96, 1990.

[2] Grzegorz Bancerek and Krzysztof Hryniewiecki. Segments of natural numbers and finite sequences. Formalized Mathematics, 1(1):107-114, 1990.

[3] Czesław Byliński. The complex numbers. Formalized Mathematics, 1(3):507-513, 1990.

[4] Czesław Byliński. Finite sequences and tuples of elements of a non-empty sets. Formalized Mathematics, 1(3):529-536, 1990.

[5] Czesław Byliński. Functions and their basic properties. Formalized Mathematics, 1(1):5565, 1990.

[6] Czesław Byliński. Functions from a set to a set. Formalized Mathematics, 1(1):153-164, 1990.

[7] Czesław Byliński. Partial functions. Formalized Mathematics, 1(2):357-367, 1990.

[8] Czesław Byliński. The sum and product of finite sequences of real numbers. Formalized Mathematics, 1(4):661-668, 1990.

[9] Agata Darmochwal. The Euclidean space. Formalized Mathematics, 2(4):599-603, 1991.

[10] Noboru Endou and Yasunari Shidama. Completeness of the real Euclidean space. Formalized Mathematics, 13(4):577-580, 2005.

[11] Krzysztof Hryniewiecki. Basic properties of real numbers. Formalized Mathematics, 1(1):35-40, 1990.

[12] Hiroshi Imura, Morishige Kimura, and Yasunari Shidama. The differentiable functions on normed linear spaces. Formalized Mathematics, 12(3):321-327, 2004.

[13] Jarosław Kotowicz. Partial functions from a domain to a domain. Formalized Mathematics, 1(4):697-702, 1990. 
[14] Jarosław Kotowicz. Real sequences and basic operations on them. Formalized Mathematics, 1(2):269-272, 1990.

[15] Beata Padlewska and Agata Darmochwal. Topological spaces and continuous functions. Formalized Mathematics, 1(1):223-230, 1990.

[16] Jan Popiołek. Real normed space. Formalized Mathematics, 2(1):111-115, 1991.

[17] Konrad Raczkowski and Paweł Sadowski. Real function differentiability. Formalized Mathematics, 1(4):797-801, 1990.

[18] Laurent Schwartz. Cours d'analyse. Hermann, 1981.

[19] Yasunari Shidama. Banach space of bounded linear operators. Formalized Mathematics, 12(1):39-48, 2004.

[20] Andrzej Trybulec. Subsets of complex numbers. To appear in Formalized Mathematics.

[21] Andrzej Trybulec. Tarski Grothendieck set theory. Formalized Mathematics, 1(1):9-11, 1990.

[22] Wojciech A. Trybulec. Pigeon hole principle. Formalized Mathematics, 1(3):575-579,

[23] Wojciech A. Trybulec. Vectors in real linear space. Formalized Mathematics, 1(2):291296, 1990.

[24] Zinaida Trybulec. Properties of subsets. Formalized Mathematics, 1(1):67-71, 1990.

[25] Edmund Woronowicz. Relations and their basic properties. Formalized Mathematics, 1(1):73-83, 1990.

[26] Edmund Woronowicz. Relations defined on sets. Formalized Mathematics, 1(1):181-186, 1990.

[27] Hiroshi Yamazaki, Yoshinori Fujisawa, and Yatsuka Nakamura. On replace function and swap function for finite sequences. Formalized Mathematics, 9(3):471-474, 2001.

[28] Hiroshi Yamazaki and Yasunari Shidama. Algebra of vector functions. Formalized Mathematics, 3(2):171-175, 1992.

Received June 6, 2007 